\title{
Effect of Vitamin B12 and folic acid supplementation on neuropsychiatric symptoms and immune response in HIV-positive patients
}

\author{
Prabha M. Adhikari, Mukta N. Chowta' ${ }^{1}$, John T. Ramapuram, Satish B. Rao, Karthik Udupa, Sahana D. Acharya ${ }^{1}$
}

Departments of Medicine and ${ }^{1}$ Pharmacology, Kasturba Medical College, Manipal University, Mangalore, Karnataka, India

\begin{abstract}
Background: Micronutrients such as B12 and folic acid deficiencies are found in higher number in HIV-infected patients. Objective: We conducted a study to examine the effect of Vitamin B12 and folic acid supplementation on neuropsychiatric manifestations, CD4 count, and anthropometric measurements in HIV-positive patients. Materials and Methods: Three different groups of HIV patients, namely, HIV patients with tuberculosis, HIV patients with neuropsychiatric manifestations, and asymptomatic HIV patients with 50 patients in each group were included in the study. Baseline and follow-up $\mathrm{CD}_{4}$ count, anthropometric measurements, neuropsychiatric assessments, Vitamin B12, and folic acid estimation were done. Results: The prevalence of folic acid deficiency was $27.1 \%$ in Group I, $31.9 \%$ in Group II, and 23.4\% in Group III. The prevalence of Vitamin B12 deficiency was 8.16\% in Group I, $6.12 \%$ in Group II, and $4.16 \%$ in Group III. HIV patients with neuropsychiatric manifestations were noted to have the lowest mean mini-mental score. After the supplementation of vitamins, anthropometric measurements, MMSE as well as Hamilton depression scores, improved in all the three groups whereas Hamilton anxiety scores improved only in Group III. The CD4 count also improved in Groups I and II after the supplementation of vitamins. Conclusion: Folic acid deficiency was highest among neuropsychiatric patients. The majority of people who had a folic acid deficiency have shown improvement in their neuropsychiatric assessment scores as well as $\mathrm{CD}_{4}$ count after its supplementation.
\end{abstract}

Key words: $\mathrm{CD}_{4}$ count, folic acid, HIV, Vitamin B12

\section{Introduction}

Micronutrients such as vitamins and mineral deficiency are associated with HIV infection and contribute to worsening of the disease and increased mortality. The prevalence of micronutrients deficiency is more in HIV-infected patients than the non-HIV individuals. HIV-positive patients appear to require an intake of these nutrients in multiples of recommended dietary allowances when compared to normal individuals. Micronutrients are essential for maintaining proper

\section{Address for correspondence:}

Dr. Mukta N. Chowta, Department of Pharmacology,

Kasturba Medical College, Manipal University,

Mangalore - 575 001, Karnataka, India.

E-mail:muktachowta@yahoo.co.in

\begin{tabular}{|l|l|}
\hline \multicolumn{2}{|c|}{ Access this article online } \\
\hline Quick Response Code: & Website: \\
\hline & www.ruralneuropractice.com \\
\hline & \\
\hline
\end{tabular}

immunologic function. ${ }^{[1]}$ A decreased plasma levels of B6, B12, A, E, and Zinc have been correlated with significant alterations in immune response and cognitive functions. Micronutrient supplementation has shown several clinical benefits to the patient, including a reduction in mitochondrial toxicity and an improvement in immune reconstitution. ${ }^{[2]}$ Vitamin B12 deficiency impairs neutrophil function and folic acid deficiency depresses the cell-mediated immunity response. Micronutrients such as B12 and folic acid deficiencies are found in higher number in HIV-infected patients. ${ }^{[3]}$ Deficiencies of these vitamins typically present initially with neuropsychiatric disturbances including depression, dementia, and demyelinating myelopathy. ${ }^{[4]}$ Although there are several studies examining the effects of

This is an open access article distributed under the terms of the Creative Commons Attribution-NonCommercial-ShareAlike 3.0 License, which allows others to remix, tweak, and build upon the work non-commercially, as long as the author is credited and the new creations are licensed under the identical terms.

For reprints contact: reprints@medknow.com

How to cite this article: Adhikari PM, Chowta MN, Ramapuram JT, Rao SB, Udupa K, Acharya SD. Effect of Vitamin B12 and folic acid supplementation on neuropsychiatric symptoms and immune response in HIV-positive patients. J Neurosci Rural Pract 2016;7:362-7. 
micronutrient supplementation in HIV-positive patients in general, there are no studies describing the effect of supplementation of specific micronutrients such as Vitamin B12 and folic acid. Hence, this study was carried out to examine the effect of Vitamin B12 and folic acid supplementation on neuropsychiatric manifestations, CD4 count, and anthropometric measurements in HIV-positive patients.

\section{Materials and Methods}

This cohort study is done at a Tertiary Care Hospital attached to a Medical College in South India. The study protocol was approved by the Institutional Ethics Committee and the written informed consent was taken from all participants before their inclusion in the study. The study consisted of three groups, each group having 50 subjects.

- Group I: HIV-positive patients with coexistent tuberculous infection

- Group II: HIV-positive patients with neuropsychiatric symptoms

- Group III: HIV-positive patients without neuropsychiatric symptoms or tuberculosis.

Definition of HIV positivity was taken as any patient tested positive by two HIV ELISA tests and a monospot test using three different antigens (or) 3 monospot test positive using three different antigens by Voluntary Counseling and Testing Centre established by the National AIDS Control Organization, India.

Neurological manifestations included strokes, central nervous system (CNS) toxoplasmosis, cryptococcal meningitis, cytomegalovirus encephalitis, and peripheral neuropathy. Psychiatric manifestations included delirium, acute confusional state, dementia, depression, and severe anxiety and mood disturbances.

Patients were inquired regarding any prior multivitamin supplementations and the duration of the same before enrolling into the study. A detailed clinical examination was performed which included detailed neurological assessment as well as psychiatric assessment.

Absolute CD4 count estimation was done in all patients using flow cytometry (normal reference range was 380-1200 cells/ $\mu \mathrm{L}$ ). Folic acid estimation was done using carbonyl metallo immunoassay method and reference range that was taken for this study was $2.7-34.0 \mathrm{ng} / \mathrm{mL}$. Vitamin B12 estimation was done using carbonyl metallo immunoassay method and reference range that was taken for this study was $189-883 \mathrm{pg} / \mathrm{mL}$. If the patient is found to have folic acid deficiency, they are supplemented with 5 $\mathrm{mg}$ in folic acid daily. If the patient was found Vitamin B12 deficient, injection Vitamin B12 was supplemented till the symptoms resolved and then once a month for 6 months.

If the patient is symptomatic, all the necessary investigations to diagnose the opportunistic infections were done and treated accordingly. Those who could not come for follow-up were considered as dropouts. Patients who were initiated antiretroviral therapy before the study or after enrollment into the study were also periodically reviewed for any side effects of the medications along with the compliance.

Follow-up of patients was done for 6 months at an interval of 2 weeks in the $1^{\text {st }}$ month followed by monthly follow-up. At each visit, $24 \mathrm{~h}$ diet recall history was obtained and subjects were counseled regarding healthy food practices and they were subjected to neuropsychiatric assessment using Folstein's Mini Mental State Examination (MMSE), ${ }^{[5]}$ Hamilton depression score (HAM-D $)^{[6]}$ and anxiety score (HAM-A), ${ }^{[7]}$ anthropometric measurements and neurological assessment for any peripheral neuropathy, etc., The immune status was assessed by CD4 count estimation. Throughout the study, the standard of care was extended to all the subjects. At the end of 6 months, patients were analyzed for any changes in the neuropsychiatric assessment scores, anthropometric parameters, and immune response as evidenced by CD4 count.

\section{Sample size}

The sample size was calculated using Open Epi Version 3 (Open Source Epidemiologic Statistics for Public Health. www.OpenEpi.com). Assuming that population folate and Vitamin B12 deficiency is 5\%, a sample size of 32 per group was calculated at a confidence level of $80 \%$ with the confidence limits as $5 \%$. Accounting for dropouts, a sample of 50 per group was included.

\section{Statistical analysis}

Statistical analysis was done using statistical package SPSS 17.3 version (SPSS Statistics for Windows, Version 17.0. Chicago: SPSS Inc.). All the continuous variables were expressed as mean or median. ANOVA was used to detect the significance of differences in means among the groups.

\section{Results}

A total of 150 patients were included in the three groups with fifty patients in each group. Among these, 74 completed the study, 33 expired, and 43 were lost to follow-up. The mean age of the patient population 
was $38.00 \pm 8.12$ years. The sex distribution included 108 males and 42 females. The median CD 4 count was 139 (58.25-330.75) cells $/ \mu \mathrm{L}$. The median duration of illness was $18.50(2,54.25)$ months, i.e. from the date of diagnosis of HIV illness to the date enrollment into the study.

Group I included HIV patients with coexistent tuberculosis. Group II included HIV-positive patients with neuropsychiatric manifestations. Group III included asymptomatic HIV patients or patients with minor opportunistic infections. Mean age of presentation for Group I, II, and III was $36.28 \pm 5.93,39.22 \pm 9.02$, and $38.57 \pm 8.84$ years, respectively, and was comparable among three groups. The ratio of males and female subjects enrolled in the three groups was 40:10, 35:15, and 33:17, respectively, which is on par with the sex ratio of HIV-positive patients.

The median baseline folic acid levels in Groups I-III were $5.91(2,9.37), 3.64(2.12,8.17)$, and $5.08(3.16,9.96) \mathrm{ng} / \mathrm{ml}$, respectively. The prevalence of folic acid deficiency in Group I was 27.1\%, Group II was 31.9\%, and Group III was $23.4 \%$. The overall prevalence of the folic acid deficiency is $27.5 \%$.

The baseline median serum B12 levels in Groups I-III were $380(263.45,701.05), 555(343,1127)$, and 460.5 $(325,698.75) \mathrm{pg} / \mathrm{ml}$, respectively. The prevalence of Vitamin B12 deficiency in Group I was $8.16 \%$, Group II was 6.12\%, and Group III was $4.16 \%$. The overall prevalence if Vitamin B12 deficiency is $7.8 \%$.

The mean follow-up body mass index (BMI) in the HIV cohort of the Groups I-III was $18.65 \pm 2.79 \mathrm{~kg} / \mathrm{m}^{2}$, $19.82 \pm 3.06 \mathrm{~kg} / \mathrm{m}^{2}$, and $19.51 \pm 3.02 \mathrm{~kg} / \mathrm{m}^{2}$, respectively [Figure 1]. The follow-up BMI increased in all the groups, suggesting that nutritional supplementation has a beneficial effect in improving the overall nutritional status of the patient. The improvement could also be due to the antiretroviral therapy (ART), which would have been introduced for some of the followed up patients.

The follow-up median triceps skinfold thickness in Groups I-III was $6(9,12), 11(6.5,15)$, and $10(7.25,14.75)$ $\mathrm{mm}$, respectively [Figure 2]. The triceps skinfold thickness increased in Groups II and III but decreased marginally in Group I, i.e., in HIV patients with tuberculosis.

The follow-up median mid-arm circumference in Groups I-III was $22.4 \pm 2.93,23.47 \pm 2.92$, and $23.87 \pm 3.83 \mathrm{~cm}$, respectively [Figure 3]. The median mid-arm circumference increased in all the diseased groups at the end of follow-up and the increase was more in Group II compared to other groups.

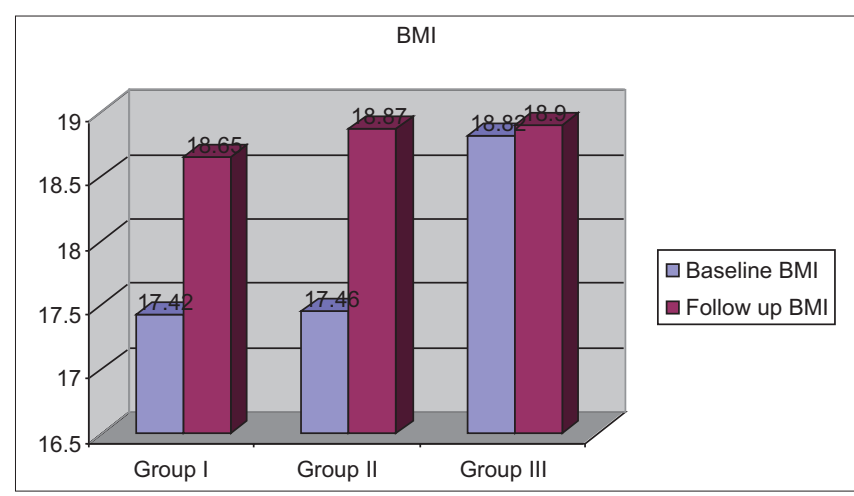

Figure 1: Changes in body mass index

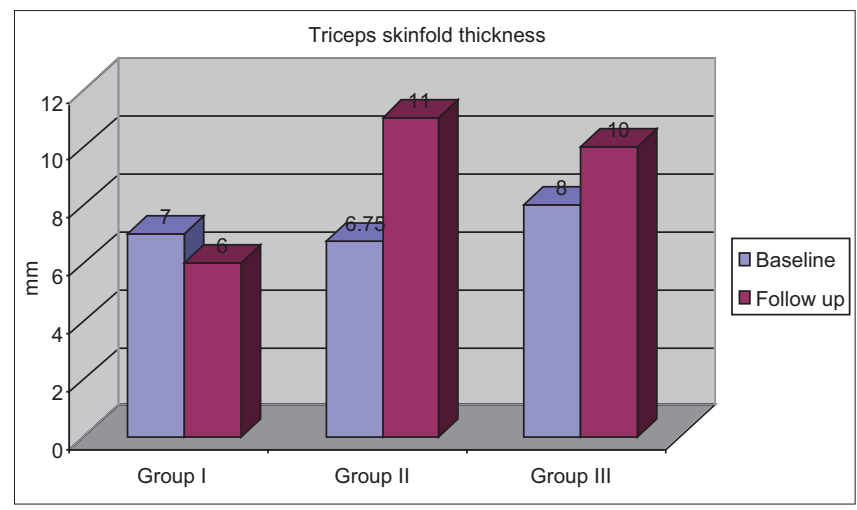

Figure 2: Changes in triceps skinfold thickness

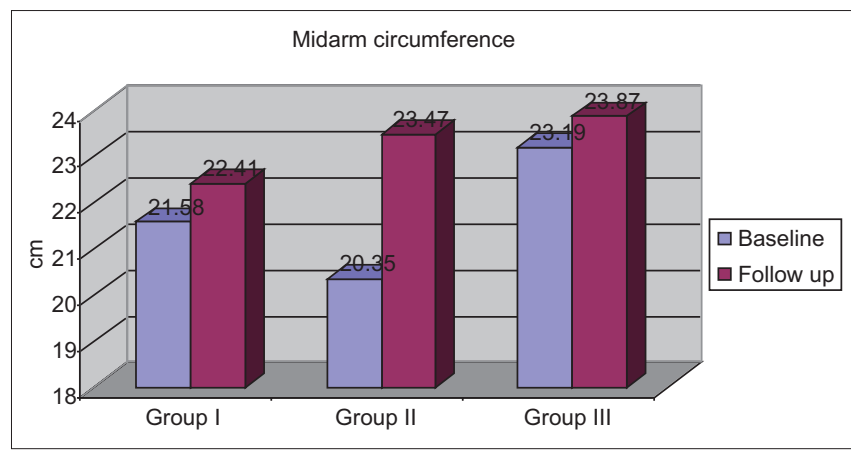

Figure 3: Changes in mid-arm circumference

The follow-up mean waist circumferencein Groups I-III was $71.78 \pm 8.07,75.68 \pm 8.82$, and $73.36 \pm 10.55 \mathrm{~cm}$, respectively [Figure 4]. The mean waist circumference increased in all the diseased groups at the end of follow-up and the increase was more in Group II compared to other groups.

The follow-up mean hip circumference in Groups IIII was $83.56 \pm 8.58,84.73 \pm 7.16$, and $86.15 \pm 7.61 \mathrm{~cm}$, respectively [Figure 5]. The mean hip circumference increased in all the diseased groups at the end of follow-up and the increase was more in Group II compared to other groups.

Table 1 shows the baseline values for mean MMSE scores, median HAM-A scores, and median HAM-D 


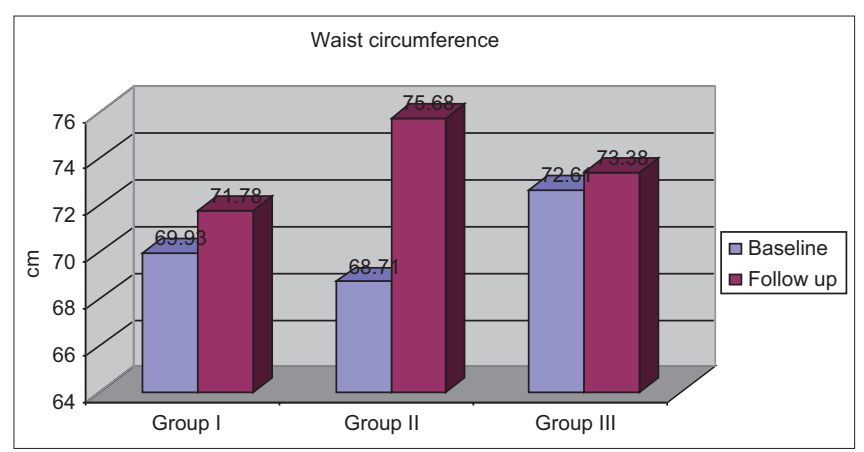

Figure 4: Changes in waist circumference

Table 1: Baseline neuropsychiatric scores

\begin{tabular}{lcccc}
\hline & Group I & Group II & Group III & $\boldsymbol{P}$ \\
\hline MMSE (mean \pm SD) & $26.98 \pm 2.63$ & $24.71 \pm 4.49$ & $27.96 \pm 2.01$ & $<0.001^{*}$ \\
Median HAM-A & $4(3,8)$ & $4(4,8)$ & $4(2,6)$ & 0.195 \\
Median HAM-D & $8(5.75,10)$ & $8(6,14)$ & $6(4,10)$ & $0.018^{*}$
\end{tabular}

${ }^{*}$ ANOVA significant. MMSE: Mini-mental score, HAM-D: Hamilton Depression Score, HAM-A: Hamilton Anxiety Score, SD: Standard deviation

scores in Groups I-III, respectively. HIV patients with neuropsychiatric manifestations (Group II) were noted to have the lowest mean MMSE score when compared to Group I and III. The mean MMSE of Group I and Group II differs $(P=0.012)$. The mean MMSE of Group III and Group II significantly differs $(P<0.001)$ [Table 1 ]. At the end of the study, MMSE scores improved in all the three groups [Figure 6]. HAM-A scores improved in Group III, but in the other two groups, it remained same [Figure 7]. HAM-D scores improved in all three groups [Figure 8].

Of 20 folic acid deficient patients who completed 6 months follow-up, 9 (45\%) improved in their HAM-A and $10(50 \%)$ improved in their HAM-D score. Of 3 Vitamin B12 deficient patients who completed 6 months follow-up, 1 (33.33\%) improved in their HAM-D score, but all three did not show improvement in HAM-A score.

Median baseline CD4 count in Groups I, II, and III was 125.5 (62.25, 271.25), 89 (46.75, 175.75), and 282 (135.50, $611.75)$ cells $/ \mu \mathrm{L}$, respectively. Statistically significant difference in the median CD4 counts $(P<0.001)$ existed between Groups I and III and also between Groups II and III $(P<0.001)$. The follow-up median CD4 count has improved in Groups I and II, but there was a marginal fall in the median CD4 count of Group III [Figure 9].

\section{Discussion}

This study has examined the effect of supplementation of Vitamin B12 and folic acid on the neuropsychiatric manifestations, immunological response, and

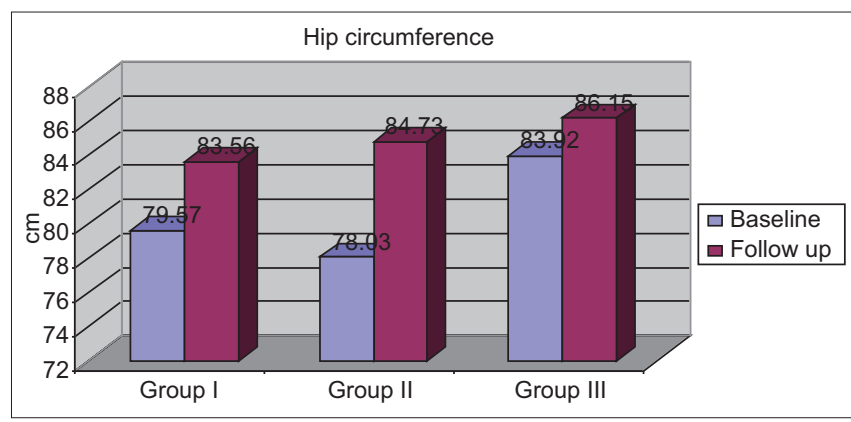

Figure 5: Changes in hip circumference

anthropometric measurements in three different groups of HIV patients, namely, HIV patients with tuberculosis, HIV patients with neuropsychiatric manifestations, and asymptomatic HIV patients. The mean age of the patient population was $38.00 \pm 8.12$ years and was comparable between these three HIV groups. In a study conducted by Jiamton et al.$^{[8]}$ on impact of multiple micronutrient supplementation on mortality among HIV-infected individuals, the median age was 32 years.

The mean BMI was more in asymptomatic HIV patients compared to the other two groups. The presence of opportunistic infections or advanced disease in Groups I and II might have led to weight loss and wasting. ${ }^{[9]}$ The mean BMI in a study conducted by Jiamton et al..$^{[8]}$ at Bangkok was 21.2 (2.7), which is higher than that found in our study. This indicates that malnutrition and wasting are more common in HIV-affected individuals in our country because of poverty, undernutrition, and late presentation due to poor awareness of the disease. All the other baseline anthropometric parameters such as mid-arm circumference, triceps skinfold thickness, and waist and hip circumference also showed the same trend.

MMSE score was lowest in Group II as they had specific neuropsychiatric problems. HAM-D scores were higher in Groups I and II compared to Group III because of the advanced nature of the disease in both groups and specific CNS involvement in Group II. High prevalence of folic acid deficiency resulting in diminished cognitive function and depression in these groups would have contributed to low mini-mental score and high HAM-D score. There was no significant difference between the groups with regard to HAM-A scores.

Median baseline CD4 count was less in Group I and II compared to Group III because of the advanced HIV disease in those two groups. The incidence of opportunistic infections, mainly affecting the CNS, leads to neuropsychiatric manifestations and is maximum in Group II because of low CD4 counts 


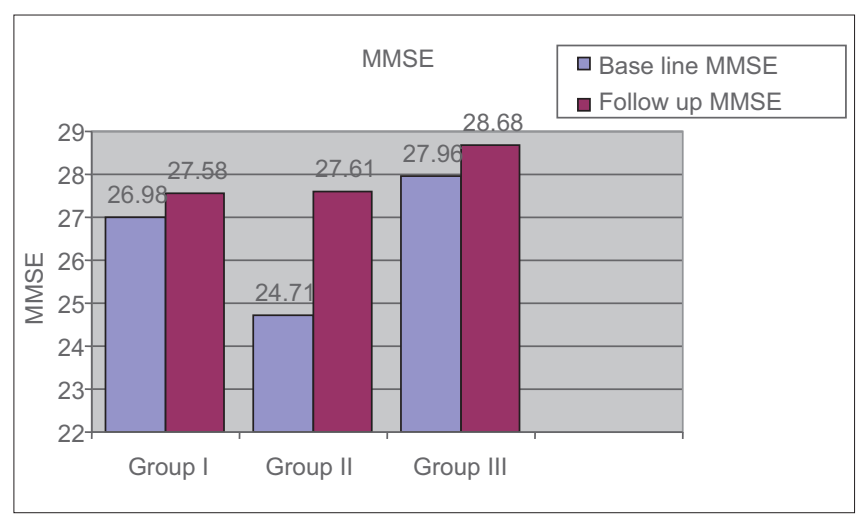

Figure 6: Changes in MMSE

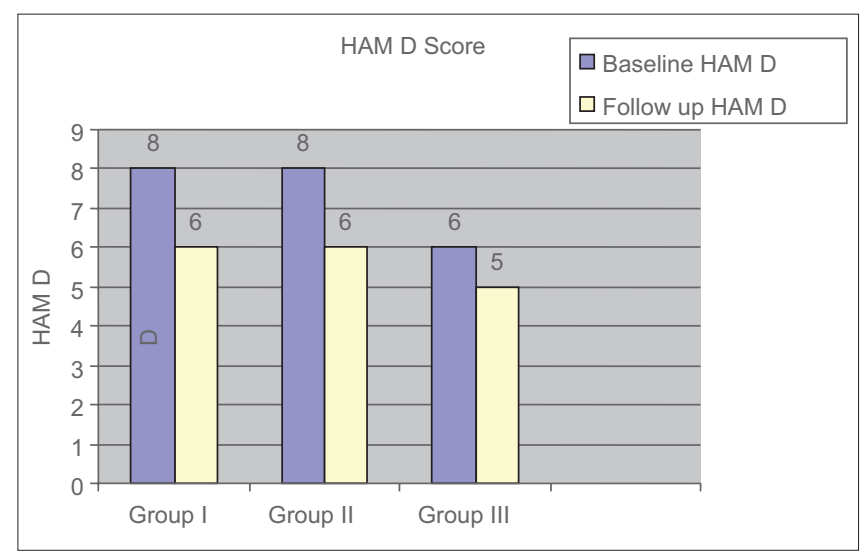

Figure 8: Changes in Hamilton depression score

and lower immune status of the patients. The median CD4 count in the study conducted by Jiamton et al. ${ }^{[8]}$ was 244, which is comparable to the median CD4 count of Group II, which was $282(135,611)$ that comprised asymptomatic HIV-positive patients. However, in a study conducted by Fawzi et al. ${ }^{[10]}$ in Tanzania on 1078 pregnant women with HIV infection, mean CD4 count was $429 \pm 224$. This might have been because of early detection of HIV infection as well as prompt initiation ART in this region.

The median folic acid level was lower in Group II compared to Group I and III, which may be because of cachexia induced malnutrition and decreased absorption of folic acid due to advanced disease in this group. Prevalence of folic acid deficiency was higher in Group II, which might have played a role in diminished cognitive function and depression found in this group. The median Vitamin B12 level was lower in Group I compared to other groups. The prevalence of Vitamin B12 deficiency was highest in Group I indicating that B12 deficiency is common in chronic diseases. The high prevalence of Vitamin B12 and folic acid deficiency in Group I indicates that special attention is required regarding micronutrient

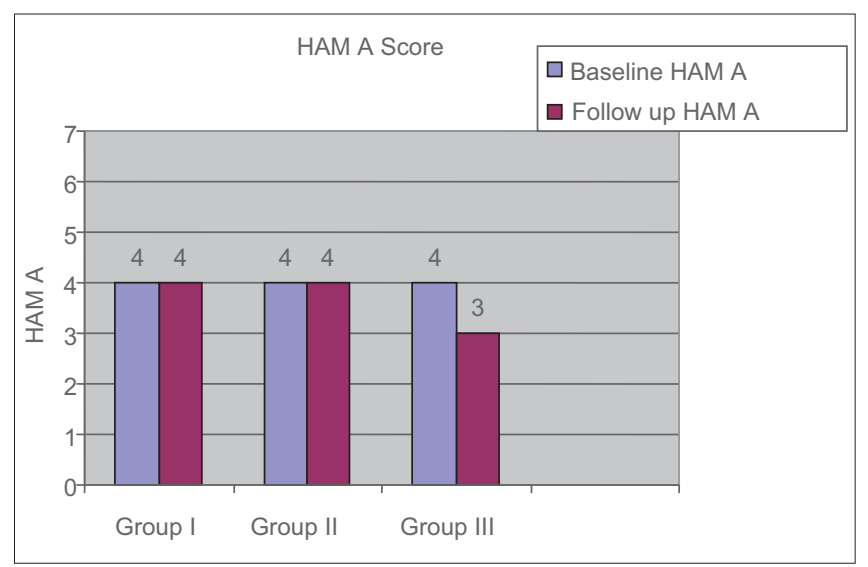

Figure 7: Changes in Hamilton anxiety score

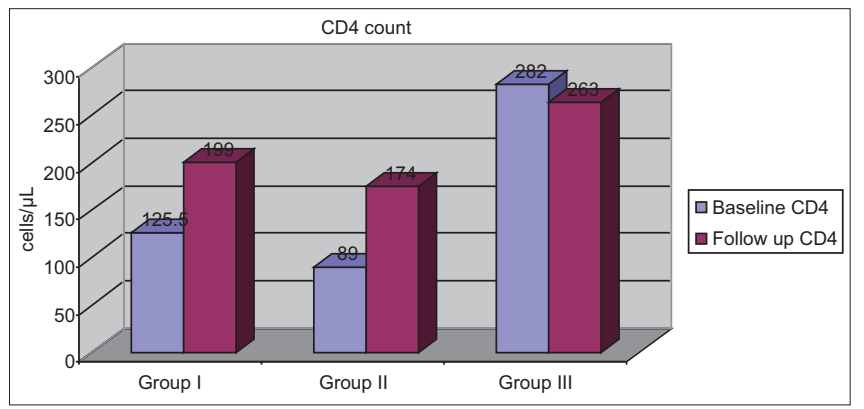

Figure 9: Changes in CD4 count

supplementation among HIV patients who have coexisting tuberculosis.

In our study, there was a significant correlation between Vitamin B12 deficiency and mortality but there was no association between folic acid deficiency and mortality. This is consistent with the study done by Tang et al., ${ }^{[11]}$ in which they found that participants with low Vitamin B12 concentration had significantly shortened AIDS-free time. In contrast, folic acid deficiency did not have any association with progression to AIDS or decline in CD4 count.

All the anthropometric parameters improved at the end of the study in all three HIV-infected groups. This may be because of appropriate nutritional supplementation and also the concomitant ART administration. The neuropsychiatric scores also improved at the end of the study in all the groups which indicates a beneficial effect of nutritional supplements on neuropsychiatric symptoms.

The median CD4 count in Group I and II increased at the end of follow-up but the median CD4 count in Group III dropped marginally. The increase in the CD4 count in the Groups II and III may be due to the nutritional supplementation and also the concomitant use of ART in these patients. The marginal fall in the CD4 count 
in asymptomatic HIV group might be because of the fact that as per NACO guidelines, ART is not indicated in this group of patients. ${ }^{[12]}$ The fall in CD4 count in these patients could be because of the continuing viral replication in the absence of ART.

Patients with folic acid deficiency were supplemented with folic acid and were improved in BMI at the end of follow-up. This signifies the role of supplementation of folic acid in deficient individuals. Of 20 folic acid deficient patients, 9 improved in HAM-A and 10 improved in HAM-D after supplementation with folic acid. This indicates that folic acid plays a role in anxiety and depression. Low levels of folic acid and Vitamin B12 have been found in studies of depressive patients, and an association between depression and low levels of the two vitamins is found in studies of the general population. Low folate level is also associated with a poor response to antidepressants, and treatment with folic acid is shown to improve response to antidepressants. ${ }^{[13]}$ However, such benefit in the improvement of HAM-A and HAM-D scores was not found with B12 supplementation in patients who had Vitamin B12 deficiency.

Patients who were found to have a folic acid deficiency improved in CD4 count after supplementation. This improvement would have been contributed by ART and antitubercular therapy. The role of Vitamin B12 in improving the immunological status cannot be commented from our study as only a few number of patients with Vitamin B12 deficiency could be followed up till the end of the study. Kaiser et al. showed that micronutrient supplementation which included Vitamin B12 and folic acid twice daily for 12 weeks significantly increased absolute CD4 cell count and mean change in CD4 cell count from baseline in HIV-infected patients taking ART. ${ }^{[14]}$

Studies have shown that though ART is associated with a decreased prevalence of opportunistic gastrointestinal diseases and incidence of malnutrition, gastrointestinal infections, and severe gastroenteritis, which alter micronutrient absorption, may persist after ART initiation. ${ }^{[15]}$ Several HIV medications, particularly nucleoside reverse transcriptase inhibitors, can inhibit the replication of mitochondrial DNA and cause vomiting and diarrhea that can reduce the absorption or increase the losses of several micronutrients. ${ }^{[16]}$

\section{Conclusion}

The prevalence of Vitamin B12 deficiency was higher among HIV patients with tuberculosis. Folic acid deficiency was highest among neuropsychiatric patients signifying its role in neuropsychiatric manifestations. CD4 count was lower in HIV patients with tuberculosis and HIV patients with neuropsychiatric symptoms. The majority of people who had a folic acid deficiency has shown an increase in their BMI, HAM-A, and HAM-D scores after supplementation. Folic acid supplementation in people with folic acid deficiency leads to improvement in CD4 count.

\section{Financial support and sponsorship \\ The grant from ICMR.}

\section{Conflicts of interest}

There are no conflicts of interest.

\section{References}

1. Cunningham-Rundles S, McNeeley DF, Moon A. Mechanisms of nutrient modulation of the immune response. J Allergy Clin Immunol 2005;115:1119-28.

2. Drain PK, Kupka R, Mugusi F, Fawzi WW. Micronutrients in HIV-positive persons receiving highly active antiretroviral therapy. Am J Clin Nutr 2007;85:333-45.

3. Tilkian SM, Lefevre G, Coyle C. Altered folate metabolism in early HIV infection. JAMA 1988;259:3128-9.

4. Issac TG, Soundarya S, Christopher R, Chandra SR. Vitamin B12 deficiency: An important reversible co-morbidity in neuropsychiatric manifestations. Indian J Psychol Med 2015;37:26-9.

5. Folstein MF, Folstein SE, McHugh PR. "Mini-mental state". A practical method for grading the cognitive state of patients for the clinician. J Psychiatr Res 1975;12:189-98.

6. Hamilton M. A rating scale for depression. J Neurol Neurosurg Psychiatry 1960;23:56-62.

7. Hamilton M. The assessment of anxiety states by rating. Br J Med Psychol 1959;32:50-5.

8. Jiamton S, Pepin J, Suttent R, Filteau S, Mahakkanukrauh B, Hanshaoworakul W, et al. A randomized trial of the impact of multiple micronutrient supplementation on mortality among HIV-infected individuals living in Bangkok. AIDS 2003;17:2461-9.

9. van Lettow M, Harries AD, Kumwenda JJ, Zijlstra EE, Clark TD, Taha TE, et al. Micronutrient malnutrition and wasting in adults with pulmonary tuberculosis with and without HIV co-infection in Malawi 2004;4:61.

10. Fawzi WW, Msamanga GI, Spiegelman D, Wei R, Kapiga S, Villamor E, et al. A randomized trial of multivitamin supplements and HIV disease progression and mortality. N Engl J Med 2004;351:23-32.

11. Tang AM, Saah NM, Graham H, Chandra RK, Alfred J. Low serum Vit B12 concentration associated with faster human immunodeficiency virus type - 1 (HIV-1) disease progression. J Nutr 1997;127:345-51.

12. Antiretroviral Therapy Guidelines for HIV Infected Adults and Adolescents. NACO. Ministry of Health and Family Welfare; May, 2013. Available from: http://www.nacoonline.org. [Last accessed on 2016 Jan 16].

13. Coppen A, Bolander-Gouaille C. Treatment of depression: Time to consider folic acid and Vitamin B12. J Psychopharmacol 2005;19:59-65.

14. Kaiser JD, Campa AM, Ondercin JP, Leoung GS, Pless RF, Baum MK. Micronutrient supplementation increases CD4 count in HIV-infected individuals on highly active antiretroviral therapy: A prospective, double-blinded, placebo-controlled trial. J Acquir Immune Defic Syndr 2006;42:523-8.

15. Miller TL. Nutritional aspects of HIV-infected children receiving highly active antiretroviral therapy. AIDS 2003;17 Suppl 1:S130-40.

16. Knox TA, Spiegelman D, Skinner SC, Gorbach S. Diarrhea and abnormalities of gastrointestinal function in a cohort of men and women with HIV infection. Am J Gastroenterol 2000;95:3482-9. 